\title{
Interaction of LRRK2 with kinase and GTPase signaling cascades
}

\author{
Joon Y. Boon ${ }^{1}$, Julien Dusonchet ${ }^{1}$, Chelsea Trengrove ${ }^{1}$ and Benjamin Wolozin ${ }^{1,2}{ }^{*}$ \\ 'Department of Pharmacology and Experimental Therapeutics, Boston University School of Medicine, Boston, MA, USA \\ ${ }^{2}$ Neurology, Boston University School of Medicine, Boston, MA, USA
}

\author{
Edited by: \\ Jean-Marc Taymans, KU Leuven, \\ Belgium \\ Reviewed by: \\ $R$. Jeremy Nichols, The Parkinson's \\ Institute, USA \\ Patrick Lewis, University College \\ London, UK \\ *Correspondence: \\ Benjamin Wolozin, Department of \\ Pharmacology and Experimental \\ Therapeutics, Boston University \\ School of Medicine, 72 East Concord \\ Street, R614, Boston, \\ MA 02118-2526, USA \\ e-mail: bwolozin@bu.edu
}

\begin{abstract}
LRRK2 is a protein that interacts with a plethora of signaling molecules, but the complexity of LRRK2 function presents a challenge for understanding the role of LRRK2 in the pathophysiology of Parkinson's disease (PD). Studies of LRRK2 using over-expression in transgenic mice have been disappointing, however, studies using invertebrate systems have yielded a much clearer picture, with clear effects of LRRK2 expression, knockdown or deletion in Caenorhabditis elegans and Drosophila on modulation of survival of dopaminergic neurons. Recent studies have begun to focus attention on particular signaling cascades that are a target of LRRK2 function. LRRK2 interacts with members of the mitogen activated protein kinase (MAPK) pathway and might regulate the pathway action by acting as a scaffold that directs the location of MAPK pathway activity, without strongly affecting the amount of MAPK pathway activity. Binding to GTPases, GTPase-activating proteins and GTPase exchange factors are another strong theme in LRRK2 biology, with LRRK2 binding to rac1, cdc42, rab5, rab7L1, endoA, RGS2, ArfGAP1, and ArhGEF7. All of these molecules appear to feed into a function output for LRRK2 that modulates cytoskeletal outgrowth and vesicular dynamics, including autophagy. These functions likely impact modulation of $\alpha$-synuclein aggregation and associated toxicity eliciting the disease processes that we term PD.
\end{abstract}

Keywords: GTPase, kinase, trafficking, cytoskeleton, actin, autophagy, cell death, dopamine

\section{INTRODUCTION}

Parkinson's disease (PD) is the most common age-related motor disorder (Hsu et al., 2010a; Ferree et al., 2012). Accumulation of aggregated $\alpha$-synuclein to form Lewy bodies is a neuropathological hallmark for PD (Lees et al., 2009). Mutations in leucine-rich repeat kinase 2, LRRK2, gene are common genetic determinants of $\mathrm{PD}$, with at least 20 different mutations identified to date causing late-onset, familial autosomal-dominant PD (Gasser et al., 2011; Greene, 2012). The most prevalent amino acid substitution mutation in LRRK2, G2019S, has been found in 1-2\% of sporadic PD cases; with sporadic PD and LRRK2-associated PD being clinically and neurochemically indistinguishable (Healy et al., 2008). These all indicate that LRRK2 has an important role in the pathogenesis of PD.

LRRK2 is a large ubiquitous cytoplasmic protein consisting of 2527 amino acids with multiple functional domains (Cookson, 2010). LRRK2 has a homolog in mammals, termed LRRK1; LRRK1 and 2 expression appear to be complementary, with LRRK2 expression peaking in development and decreases rapidly after birth, while LRRK2 expression increases postnatally (SimonSanchez etal., 2006). The LRRK2 protein consists of kinase, Ras-of-complex proteins (ROCs) GTPase, C-terminal of ROC (COR), leucine-rich repeat, ankyrin and WD40 domains (Cookson, 2010). The two catalytic domains are the kinase and ROC GTPase domains. The kinase domain has the highest sequence homology to mitogen-activated protein kinase kinase kinase (MKKK)/MLKs (mixed lineage kinases) and receptor-interacting protein (RIP) kinase families. The most common mutation in LRRK2 associated with PD is the G2019S mutation located in the kinase domain of LRRK2 (Wszolek et al., 2004; Zimprich et al., 2004). Other common amino acid substitution include $\mathrm{R} 1441 \mathrm{C} / \mathrm{G} / \mathrm{H}$ in the Roc GTPase domain and Y1699C in the COR domain (Taylor et al., 2006). The pathological mutations G2019S enhances the kinase activity without affecting the GTPase activity of LRRK2; whereas R1441C/G/H and Y1699C affect GTPase activity of LRRK2 by impairing GTP hydrolysis and has inconsistent effects on its kinase activity (West et al., 2005; Greggio et al., 2006; Ito et al., 2007; Lewis et al., 2007; Li et al., 2007). However, the underlying mechanisms linking such mutations to pathology still remain unclear.

\section{LRRK2 MODULATES SURVIVAL OF DOPAMINERGIC NEURONS, AUTOPHAGY, AND NEURONAL OUTGROWTH}

Studies of LRRK2 using over-expression in transgenic mice have been disappointing, with few studies showing consistent effects occurring upon over-expression of wildtype or mutant LRRK2 (Dawson et al., 2010). Knockout of LRRK2 in the mouse yielded a strong phenotype in the kidney pointing to a role for LRRK2 in autophagy, however, no clear differences in the brain; this suggests that mammalian LRRK2 in vivo models do a better job of modeling cellular function/dysfunction rather than providing a more general model of PD (Tong et al., 2010). In contrast, over-expression studies using invertebrate systems have yielded much clearer results. Orthologs of LRRK2 also exist in Drosophila 
and Nematodes, although these species exhibit just one form of LRRK, referred to as lrk-1 (Marin, 2008). The invertebrate LRRK2 orthologs have kinase, COR and ROC domains that are homologous to mammalian LRRK2, and the lrk-1 protein appears to subsume the functions of both LRRK1 and LRRK2 (Marin, 2008; Saha etal., 2009). Studies from several groups examining the effects of LRRK2 expression, knockdown or deletion in Caenorhabditis elegans and Drosophila all show strong effects (Liu et al., 2008; Wang et al., 2008; Saha et al., 2009; Samann et al., 2009; Venderova et al., 2009; Yao et al., 2010; Yuan et al., 2011). The results appear to differ somewhat based on the system, but the effects are strong in all cases. Studies in our laboratory indicate that expressing WT LRRK2 promotes survival of dopaminergic neurons in response rotenone treatment, while mutant LRRK2 (G2019S or R1441C) enhances loss of dopaminergic neurons relative to WT (Saha et al., 2009). Another important aspect of our observations, which becomes important when considering studies of LRRK2 more generally, is that G2019S also potentiates loss of neuronal processes relative to WT LRRK2. Because nematodes lack endogenous $\alpha$-synuclein, we recently crossed the LRRK2 lines to a line expressing $\alpha$-synuclein in dopaminergic neurons. Interestingly, current studies with a new nematode model in our laboratory shows that both WT and G2019S LRRK2 potentiate age-related loss of dopaminergic neurons (and of their processes), suggesting that understanding the interaction between LRRK2 and $\alpha$-synuclein is important for modeling the pathophysiology of PD. Studies from the Chen laboratory using a different C. elegans model (without incorporation of $\alpha$-synuclein) show a deleterious effect of WT and G2019S LRRK2, while studies using Drosophila indicate that LRRK2 potentiates degeneration of retinal cells and loss of dopaminergic neurons (Liu et al., 2008; Yao et al., 2010). Thus, the specific results depend on the particular model used, but it is clear that invertebrate models are very sensitive to modulation of LRRK2 levels, and mutations in LRRK2 potentiate degeneration of dopaminergic neurons. While mouse models are not particularly sensitive to LRRK2 over-expression, knockout of LRRK2 appears to potentiate autophagic deficits in the kidney, which is a site that normally exhibits strong expression of LRRK2. This provides evidence that the effects observed with invertebrate systems have strong relevance to the mammalian system.

Studies using primary rodent neurons grown in culture have yielded much stronger results than the transgenic mouse models. These studies consistently indicate that expression of mutant LRRK2 potentiates neurodegeneration, and reduces neurite outgrowth (Smith et al., 2005; Chan et al., 2011; Skibinski et al., 2014). Interestingly, acute expression of WT LRRK2 appears to be beneficial, which parallels observations from our studies in C. elegans. One study, using in utero electroporation of LRRK2 in the rodent brain also observed detrimental effects of G2019S LRRK2 and beneficial effects of WT LRRK2, suggesting that acute expression of LRRK2 in the rodent brain might produce clearer results than chronic over-expression, such as occurs with transgenic mice (Macleod et al., 2006). Taken together, these results indicate that mutant LRRK2 is detrimental; the effects of WT LRRK2 are less clear, with some data suggesting that WT LRRK2 is beneficial, and other data suggesting that over-expression can be mildly detrimental. In general, most data suggest that loss of LRRK2 expression is detrimental.

\section{LRRK2 REGULATES MAP KINASE SIGNALING PATHWAYS}

Incorporating research from multiple different venues suggests roles for LRRK2 in two differing cellular networks. Prior studies from our laboratory, and subsequent studies from the Kahle laboratory indicate that LRRK2 interacts with the mitogen activated protein kinase (MAPK) pathways (Carballo-Carbajal et al., 2010; Hsu et al., 2010a,b). The MAPK pathway is a signaling network that begins with membrane signaling and extends through multiple successive kinases leading ultimately to phosphorylation of transcription factors that act on gene expression (Yang et al., 2013). Receptor signaling frequently begins with MAP Kinase kinase kinases (MAPKKKs, also termed mixed lineage kinases, MLKs), which are enzymes often act to initiate signaling cascades that ultimately lead to transcriptional regulation (Yang et al., 2013). The MLK family of kinases activates the c-jun NH2-terminal kinase (JNK) pathway by phosphorylating MKK4 and -7 and activates the p38 pathway by phosphorylating MKK3 and -6 (Gallo and Johnson, 2002; Wang et al., 2004). These stress kinase complexes required scaffold proteins in the regulation of their subcellular localization. In particular for the MAP kinase signaling cascades, the JNK-interacting proteins (JIPs) are the group of scaffold proteins in such regulation (Kelkar et al., 2005; Whitmarsh, 2006). Hence, these MKKs are then recruited into a multi-protein complex by scaffold proteins JIPs (Habig et al., 2013). JIP1-3 regulates the specificity and localization of the JNK pathway and JIP4 is a specific scaffolding protein for the p38 pathway (Verhey et al., 2001; Kelkar et al., 2005; Whitmarsh, 2006).

Multiple studies show that LRRK2 interacts with the MAPK pathway and has a kinase domain that is homologous to MAPKKK/MLKs and RIPs (West et al., 2005; Greggio and Cookson, 2009; Carballo-Carbajal et al., 2010; Hsu et al., 2010a,b). However, these same studies suggest that LRRK2 is not a strong activator of the pathway. Our laboratory has shown that LRRK2 binds MKK6, -3, and -7 in HEK293FT (Gloeckner et al., 2009; Hsu et al., 2010a). Binding of LRRK2 to MKK6, -3, and -7, activates the p38 and JNK pathway, but the amount of activation is strikingly modest (Hsu et al., 2010a). Receptor activation of the MAPK pathway produces many fold increases in the activities of the downstream kinases, but over-expressing LRRK2 increases activities of the kinases and downstream transcription factors by $60 \%$ or less (Hsu et al., 2010a). Studies from the Kahle laboratory showed similarly low levels of kinase activation upon LRRK2 over-expression (Carballo-Carbajal et al., 2010). These studies showed that LRRK2 upregulates alphasynuclein transcription in parallel with MEK/ERK activation. This induction of transcriptional upregulation of alpha-synuclein was suppressed by treatment with the selective MAPK/ERK kinase inhibitor, U0126 (Carballo-Carbajal et al., 2010). Disease linked mutations increased activation by only an additional 25-30\%. Similar results were observed upon analysis of JIP proteins, which act as scaffolds for MAPKs, and function in transport of MAPKs (Hsu etal., 2010b). We have also shown that LRRK2 binds to JIP1-4 and is associated with increased levels of total JIP1, -3, -4, oligomeric JIP, and ubiquitinated JIP. In addition, 
G2019S, R1441C and I2020T (but not Y1699C) mutations in LRRK2 increased binding with MKK6 and also levels of JIP4 (Hsu et al., 2010b). The stimulation of JIP oligomerization was particularly striking effect observed upon co-expression of LRRK2 with JIPs.

Despite the relatively modest effect of LRRK2 on the MAPK cascades, LRRK2 produces a robust effect in vivo, and the MAPKs appear to be necessary for this function. Our in vivo studies in $C$. elegans showed robust protection against mitochondrial stress that was induced by LRRK2 expression. This protection was dependent on the action of MKK6 or p38 because RNAi knockdown of endogenous orthologs of MKK6 or P38 (sek-1 and pmk-1) or deletion of sek-1 in C. elegans abolish neuroprotection by LRRK2 (Hsu et al., 2010a). The MAPK cascade also appears to modulate the effects of LRRK2 on autophagy (Plowey et al., 2008). Using redox proteomics, we also demonstrated that LRRK2 regulates proteins associated with the lysosome, including ATPVA6 (Di Domenico et al., 2012). Application of a MAPKK (MEK) inhibitor blocked the effects of LRRK2 on autophagy and on neurite shortening (Plowey et al., 2008). Thus, interactions between LRRK2 and members of the MAPK pathway cause significant physiological effects despite causing only modest changes in phosphorylation.

The discordance between the modest effects of LRRK2 on activation of the MAPK pathway and the strong effects of LRRK2 in protecting dopaminergic neurons in the nematode, or the strong effect of LRRK2 on JIP oligomerization demands a novel model to explain LRRK2 function. One possibility is that LRRK2 acts as a scaffold that directs the location of MAPK pathway activity, without strongly affecting the amount of MAPK pathway activity. Location is as important to enzymatic action as amount of activity. JIPs for instance, are thought to function in vesicular trafficking. If LRRK2 also exhibited a role in vesicular trafficking, then it could impact on neuronal function by directing particular enzymes toward trafficked vesicles, rather than explicitly activating the MAPK pathway. Such a role would also be consistent with other studies suggesting a function for LRRK2 in regulating small GTPases associated with vesicles.

\section{LRRK2 REGULATES MANY OTHER SIGNALING CASCADES}

Recent transcriptome and proteome studies emphasize the diverse number of pathways regulated by LRRK2. Studies by the Dawson group recently highlighted regulation of translational functions by LRRK2, showing that LRRK2 binds to and phosphorylates the ribosomal protein RPS15 (Martin et al., 2014). This observation fits well with other studies showing roles for LRRK2 as a negative mediator of miR mediated translational repression (Gehrke et al., 2010). The regulatory network that we developed also identifies RPS15 as a primary member of the LRRK2 regulatory network, interacting with LRRK2 in a pathway that includes the ADP-ribose polymerase TNKS (Dusonchet et al., 2014). The Cookson group also recently published a proteomic study of LRRK2, which identified cyclin-G associated kinase (GAK) as a strong binding protein (Beilina et al., 2014). GAK is the third strongest genetic risk factor for PD, after synuclein and tau (Pankratz et al., 2009; Dumitriu et al., 2011).

Other studies identify LRRK2 as a negative regulator of PKA signaling, acting through direct interaction with PKAR11B, modulating neuronal development and function, by influencing dopamine signaling and synaptogenesis (Parisiadou et al., 2014). The R1441C LRRK2 mutation exhibits similar neuronal effects on LRRK2 knockout mice, causing dopamine signaling impairments as well as neurite retraction via enhanced PKA activation. PKA also regulates LRRK2 by phosphorylating S1444 in the ROC domain; 14-3-3 then binds to the phospho-serine 1444 and inhibits LRRK2 kinase activity (Muda et al., 2014). Binding of 14-3-3 to different phospho-epitopes (Ser-910/935) appears to stabilize LRRK2, while reduced binding destabilizes LRRK2 and causes aggregation of LRRK2 to form cytoplasmic inclusions (Dzamko et al., 2010). Identification of targets of LRRK2 kinase activity remains unclear. Studies have identified numerous putative substrates, including MAPK, 4E-BP, and Tau (Imai et al., 2008; Berwick and Harvey, 2011; Bailey et al., 2013).

Although LRRK2 has gained the most attention for its putative role in modulating dopamine function, LRRK2 also exerts regulation over immune responses. LRRK2 inhibits the transcription factor NFAT, which plays important roles in immune function as well as inflammatory bowel disease (Liu et al., 2011). Upon overexpression of LRRK2, NFAT remains cytosolic, and is unable to translocate into the nucleus, which suppresses its transcriptional activity (Liu et al., 2011). The MyD88 pathway is an additional pathway that is associated with the role of LRRK2 in immune function. Upon inflammation, TLR signaling via the MyD88 pathway leads to phosphorylation of LRRK2, implicating LRRK2 in macrophage biology (Dzamko et al., 2012).

\section{LRRK2 REGULATES SMALL GTPases}

A repeating theme in LRRK2 biology is its interactions with small GTPases. The ROC domain in LRRK2 is striking because it forms homo- and hetero- dimers with LRRK2 and LRRK2, respectively, in vitro (Shin et al., 2008; Hsu et al., 2010a; Kumar et al., 2010). The tendency of the LRRK2 ROC domain to dimerize appears to belie a broader biological characteristic, which is an ability to bind multiple small GTPases. As described below, we have shown that LRRK2 binds the small GTPase, racl (Chan et al., 2011). This observation parallels other studies showing that LRRK2 binds to or is functionally dependent on other small GTPases, such as rab5, rab7L1 and endoA (Matta et al., 2012; Beilina et al., 2014). Additionally, LRRK2 binds multiple GTP modulating proteins (described in following sections). In the case of rac1, the interactions of LRRK2 appears to regulate the site of action of the small GTPase, and leads to strong effects on the cytoskeleton, and for arfGEF7, they regulate the growth cone (Habig et al., 2013).

The interaction between LRRK2 and rac1 was observed by coimmunoprecipitation. LRRK2 could immunoprecipitate with rac1 upon over-expression in cell lines (HEK293FT cells), as well as from endogenous human brain striatum; in contrast binding to Cdc42 was weak and binding to RhoA was not apparent (Chan et al., 2011). Specificity of the interaction was shown by selective precipitation of $\mathrm{racl}$, without precipitation of the other classic Rho GTPases - Cdc42 and RhoA, although another study did find evidence for interaction of LRRK2 with cdc42 (Chan et al., 2011; Habig et al., 2013). The interaction between LRRK2 and rac1 occurs through the ROC-COR kinase domains. Co-expressing LRRK2 and racl enhanced racl activity by increasing binding 
of racl to p21-activated kinase, which in turn modulates actin cytoskeletal dynamics. LRRK2 with inactivated kinase or GTPase domains does not activate racl (Chan et al., 2011). LRRK2 does not increase membrane-bound racl, but it significantly changes the cellular localization of rac1, causing polarization, which is further augmented when LRRK2 is co-expressed with constitutively active rac1. G2019S and R1441C LRRK2 mutations decrease rac1 binding; whereas Y1699C and I2020T increase rac1 binding.

Rac1 is known to play an important role in actin cytoskeleton remodeling that is required for the maintenance of neurite morphology (Chan et al., 2011). The interaction between LRRK2 and racl results in distinct effects associated with changes in the actin cytoskeleton. Previous studies have shown that G2019S induces neurite retraction in both in vitro and in vivo studies through the MAPK signaling pathway and such pathology precedes dopaminergic neuronal death by apoptosis (Liou et al., 2008; Plowey et al., 2008; Parisiadou et al., 2009; Carballo-Carbajal et al., 2010; Hsu et al., 2010a,b). In SH-SY5Y cells, co-expression of rac1 and G2019S has shown to rescue neurite retraction induced by G2019S. These studies suggest that mutations in LRRK2 can lead to a decrease in activation of rac1, which causes disassembly of actin filaments leading to neurite retraction (Chan et al., 2011).

\section{SYSTEMS BIOLOGY PROVIDES A COMPREHENSIVE ASSESSMENT OF CELLULAR PATHWAYS INTERACTING WITH LRRK2}

The studies showing LRRK2 interacting with many varied cellular proteins present a challenge for understanding its function. LRRK2 has been suggested to bind to many different proteins, including moesin, tubulin, MKK3, 6 and 7, JIP1, 3, and 4, arfGAP1, arhGEF7, endoA, cyclin-GAK, rab5, rab7L1, 14-3-3, (Imai et al., 2008; Shin et al., 2008; Ko et al., 2009; Sancho et al., 2009; Gehrke et al., 2010; Hsu et al., 2010a,b; Kumar et al., 2010; Nichols et al., 2010; Chan et al., 2011; Matta et al., 2012; Stafa et al., 2012; Habig et al., 2013; Beilina et al., 2014). LRRK2 has a regulatory role in a wide variety of biological processes; such as, protein translations, cytoskeletal processes, vesicular dynamics, neurite extension, mitochondrial function, endoplasmic reticulum function, and autophagy. Broad proteomics studies point to interactions with multiple new proteins, as well as regulation of many mitochondrial and lysosomal proteins (Di Domenico et al., 2012; Beilina et al., 2014). The multiple functions and pathways associated with LRRK2 suggest a complex role for LRRK2 in neuronal biology. We have approached this question by using systems biology to create a regulatory network that outlines the multiplicity of functional interactions of LRRK2 with its partners.

We employed an in silico approach to elucidate the gene regulatory network linked to LRRK2 (Dusonchet et al., 2014). This approach used a network algorithm, termed the context likelihood of relatedness (CLR; Faith et al., 2007). This algorithm is designed to analyze state-dependent genome-wide expression data based on the degree of synchrony in variation of transcript levels among samples. Thus, transcripts whose expression varies in concert with LRRK2 transcripts are deemed neighbors. We analyzed both brain and white blood cells; use of white blood cells diversified the pathway representation away from a predominance of genes linked to cell death pathways, presumably because white blood cells are not post-mortem tissues. The LRRK2-centered association subnetwork identified many known interactors, as well as many novel linked pathways (Dusonchet et al., 2014). For instance, network proteins that have been previously associated with LRRK2 are present in this network, including DJ-1, PINK1, MKK7, and JIP1 (Ho et al., 2009; Sancho et al., 2009; Carballo-Carbajal et al., 2010; Hsu et al., 2010a,b; Ferree et al., 2012; Dusonchet et al., 2014). The network also identified many novel networks linked to LRRK2. Subsequent studies validated the putative roles of these interacting proteins using knockdown studies in C. elegans.

Previous work from the laboratory established that LRRK2 expression protects dopaminergic neurons against degeneration induced by the mitochondrial toxin, rotenone. We tested the action of the putative network partners in LRRK2 mediated protection of dopaminergic neurons, and identified about 280 genes whose knockdown modified the effects of LRRK2 on dopaminergic neurons. Genes whose knockdown impaired LRRK2 action included genes known to be part of the LRRK2 network (DJ1, PINK1, MKK7, and JIP1), as well as multiple other genes, including HDAC6, vps34 and unc51, each of which is associated with autophagy (Dusonchet et al., 2014). The connection between LRRK2 and autophagy is consistent with several other prior studies, as well as with studies suggesting a role for LRRK2 in vesicular biology (Biskup et al., 2006; Plowey et al., 2008; Alegre-Abarrategui et al., 2009).

One particularly interesting proteins identified through the network analysis was the signaling gene RGS2 (regulator of G protein signaling 2), which encodes for a GTPase-activating protein (GAP), as a statistically significant regulatory "hub" in the pathways linking LRRK2 with DJ-1, PINK1, and Parkin (Dusonchet et al., 2014). RGS2 is also of particular interest because prior studies indicate strong expression in dopaminergic neurons. This positioning of RGS2 as a "hub" for multiple genes linked to PD suggests a key role for RGS2 as a regulator of LRRK2 activity, function and neuronal toxicity (Dusonchet et al., 2014). RGS proteins are a family of proteins characterized by a GAP domain of $\sim 130$ amino acids, the RGS domain. Other GAP proteins have also been associated with LRRK2 function, including ArfGAP1 and ArhGEF7 (Stafa et al., 2012; Xiong et al., 2012; Habig et al., 2013). Although GAPs and GTPase exchange factors (GEFs) are classically considered to function in regulating signaling of G-protein coupled receptors, these GAPs exhibit a strong role in regulating LRRK2 GTPase activity. Recombinant RGS2 and ArhGEF7 increase the GTPase activity of immunopurified fulllength LRRK2 in a dose-dependent manner in vitro. Recombinant RGS2 also inhibits the LRRK2 kinase activity in a dose-dependent manner. However, the concentration of RGS2 required to achieve maximal inhibition of LRRK2 kinase activity, occurs at one tenth of the concentration of RGS2 that is required to stimulate GTPase activity maximally. It is increasingly clear that the output of LRRK2 is modulated by the particular GAP or GEF bound to it. All of the GAPs and GEFs appear to increase LRRK2 GTPase activity, but RGS2 and ArhGEF7 reduce LRRK2 kinase activity, while ArfGAP1 increases LRRK2 autophosphorylation (Stafa et al., 2012; Habig et al., 2013; Dusonchet et al., 2014). This suggests that the output from the GTPase domain is determine, in 
part, by the particular GAP or GEF with which it is associated (Figure 1).

\section{GAPs AND GEFs MODULATE CYTOSKELETAL EFFECTS OF LRRK2}

A putative role for LRRK2 in regulating cytoskeletal function consistently appears in the literature. LRRK2 has been observed to regulate neurite outgrowth, tubulin and microtubules, trafficking proteins and actin (Hsu et al., 2010b; Chan et al., 2011; Caesar etal., 2013; Law etal., 2014). Our studies suggest that RGS2 inhibits the tendency of LRRK2 (WT and G2019S) to reduce length and complexity of neuronal processes in primary neurons (Figure 1; Dusonchet et al., 2014). Co-expression of RGS2 with LRRK2 significantly protects against G2019S LRRK2induced neurite shortening, although RGS2 alone has no effect on axonal length. ArhGEF7 also increases LRRK2 GTPase activity but induces LRRK2 to increase neuronal arborization, length and growth cone formation (Figure 1; Habig et al., 2013). In contrast, knockdown of ArfGAP1 protects against G2019S LRRK2-induced neurite shortening (Figure 1; Stafa et al., 2012). ArhGEF7 acts through the actin cytoskeleton, which raises the possibility that it might act in tandem with racl and cdc42, both of which bind LRRK2 (Chan etal., 2011; Habig et al., 2013). These data suggest that small GTPases and their regulatory proteins act to regulate the actions of LRRK2 on the cytoskeleton and neurite outgrowth (Figure 1). Redundant actions of RGS2 and ArhGEF7 toward neurite cytoskeletal activity seems unlikely, which raises the possibility that RGS2 and ArhGEF7 exhibit divergent effects on LRRK2 when examining other functions, such as vesicular biology.

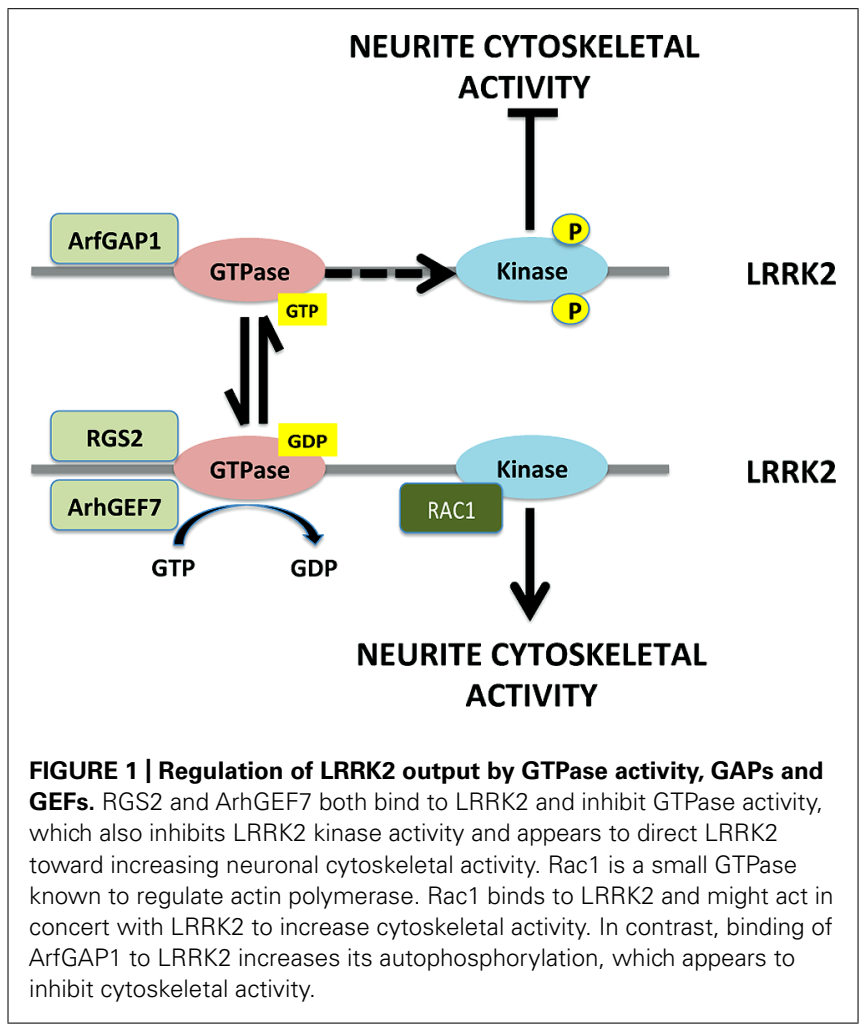

\section{CONCLUSION}

LRRK2 is a large molecule with many molecular interactions. Multiple studies have focused on its kinase activity, with the resulting identification of LRRK2 inhibitors that might have therapeutic benefit. The function of LRRK2 kinase activity remains unclear, with LRRK2 itself being the most robust substrate identified to date. Meanwhile, LRRK2 has been shown to interact with many different proteins, suggesting that important function of LRRK2 might lie in domains linked indirectly or not at all to its kinase function. A recent study suggests that an important action of phosphorylation is regulation of LRRK2 degradation, which would imply that one must look beyond kinase function to understand the role of LRRK2 in the neuron (Skibinski et al., 2014). The need to look beyond kinase function is emphasized by examination of the actions of GAPs and GEFs on LRRK2, where proteins that exhibit seemingly similar actions toward LRRK2 kinase activity elicit opposite actions on regulation of neurite outgrowth and cytoskeletal function (Figure 1).

Studies from our laboratory and other laboratories have identified numerous other signaling molecules that interact with LRRK2. The interactions of LRRK2 are clearly pleiotropic, and vary depending on the cell type and the function being investigated. Despite this complexity, several distinct themes are evolving, and these themes potentially have important implications for our understanding of the pathophysiology of PD. One theme that arises is the role of LRRK2 in regulating signaling cascades, such as the MAPK cascade. It is clear that LRRK2 acts in a manner that is different than classic modulators of the MAPK cascade, because it does not directly stimulate phosphorylation of MAP Kinases. The large size of LRRK2 enables it to bind multiple different proteins, and potentially bring them together as a signaling scaffold, analogous to JIPs.

A general theme occurring throughout the field of PD is regulation of vesicular transport. The vast majority of genes associated with PD have some function related to membranes and vesicles. $\alpha$-Synuclein is thought to regulate production of small vesicles by promoting membrane curvature (Perlmutter et al., 2009; Varkey et al., 2010; Ducas and Rhoades, 2012). LRRK2 also shows strong interactions with membranes and is thought to modulate autophagy, which also involves vesicular dynamics. PINK1, parkin, and HTRA2 are all proteins that regulate mitochondrial function, possibly including mitophagy (Plun-Favreau et al., 2007; Narendra et al., 2010). ATP13A2 and GBA are both associated with lysosomes (Mazzulli et al., 2011; Usenovic et al., 2012). The consistent appearance of vesicular biology in the pathophysiology of PD suggests that interactions of LRRK2 with vesicles are likely to contribute to its mechanism of disease pathogenesis. Future studies will need to specifically investigate how signaling pathways regulate the interactions of LRRK2 with membranous organelles, such as autophagosomes. In this context, the preliminary data showing roles for the MAPK cascade in regulating the interactions of LRRK2 with the autophagic system are intriguing (Plowey et al., 2008).

The complexity of LRRK2 signaling provide insight into the nature of pathology associated with LRRK2-mediated disease. Most cases with LRRK2 mutations exhibit $\alpha$-synuclein pathology, such as Lewy bodies, but some cases exhibit tau pathology 
(Devine and Lewis, 2008). This pathological heterogeneity might derive from the impact of different disease processes on LRRK2 biology, with some signaling cascades (such as MAPKs) promoting tau pathology and other signaling cascades (such as vesicular biology) promoting $\alpha$-synuclein pathology.

\section{AUTHOR CONTRIBUTIONS}

Joon Y. Boon drafted the manuscript, Chelsea Trengrove helped with revisions, and Benjamin Wolozin edited the manuscript. Julien Dusonchet provided important concepts for the manuscript.

\section{ACKNOWLEDGMENTS}

This work was supported by grant award to Benjamin Wolozin (NIH grants ES020395, NS066108, NS073679, NS060872, the BrightFocus Foundation, the CurePSP Foundation and the Alzheimer Association).

\section{REFERENCES}

Alegre-Abarrategui, J., Christian, H., Lufino, M. M., Mutihac, R., Venda, L. L., Ansorge, O., et al. (2009). LRRK2 regulates autophagic activity and localizes to specific membrane microdomains in a novel human genomic reporter cellular model. Hum. Mol. Genet. 18, 4022-4034. doi: 10.1093/hmg/ddp346

Bailey, R. M., Covy, J. P., Melrose, H. L., Rousseau, L., Watkinson, R., Knight, J., et al. (2013). LRRK2 phosphorylates novel tau epitopes and promotes tauopathy. Acta Neuropathol. 126, 809-827. doi: 10.1007/s00401-013-1188-4

Beilina, A., Rudenko, I. N., Kaganovich, A., Civiero, L., Chau, H., Kalia, S. K., et al. (2014). Unbiased screen for interactors of leucine-rich repeat kinase 2 supports a common pathway for sporadic and familial Parkinson disease. Proc. Natl. Acad. Sci. U.S.A. 111, 2626-2631. doi: 10.1073/pnas.1318306111

Berwick, D. C., and Harvey, K. (2011). LRRK2 signaling pathways: the key to unlocking neurodegeneration? Trends Cell Biol. 21, 257-265. doi: 10.1016/j.tcb.2011.01.001

Biskup, S., Moore, D. J., Celsi, F., Higashi, S., West, A. B., Andrabi, S. A., et al. (2006). Localization of LRRK2 to membranous and vesicular structures in mammalian brain. Ann. Neurol. 60, 557-569. doi: 10.1002/ana.21019

Caesar, M., Zach, S., Carlson, C. B., Brockmann, K., Gasser, T., and Gillardon, F. (2013). Leucine-rich repeat kinase 2 functionally interacts with microtubules and kinase-dependently modulates cell migration. Neurobiol. Dis. 54, 280-288. doi: 10.1016/j.nbd.2012.12.019

Carballo-Carbajal, I., Weber-Endress, S., Rovelli, G., Chan, D., Wolozin, B., Klein, C. L., et al. (2010). Leucine-rich repeat kinase 2 induces alpha-synuclein expression via the extracellular signal-regulated kinase pathway. Cell Signal. 22, 821-827. doi: 10.1016/j.cellsig.2010.01.006

Chan, D., Citro, A., Cordy, J. M., Shen, G. C., and Wolozin, B. (2011). Rac1 protein rescues neurite retraction caused by G2019S leucine-rich repeat kinase 2 (LRRK2). J. Biol. Chem. 286, 16140-16149. doi: 10.1074/jbc.M111.234005

Cookson, M. R. (2010). The role of leucine-rich repeat kinase2 (LRRK2) in Parkinson's disease. Nat. Rev. Neurosci. 11, 791-797. doi: 10.1038/nrn2935

Dawson, T. M., Ko, H. S., and Dawson, V. L. (2010). Genetic animal models of Parkinson's disease. Neuron 66, 646-661. doi: 10.1016/j.neuron.2010. 04.034

Devine, M. J., and Lewis, P. A. (2008). Emerging pathways in genetic Parkinson's disease: tangles, Lewy bodies and LRRK2. FEBS J. 275, 5748-5757. doi: 10.1111/j.1742-4658.2008.06707.x

Di Domenico, F., Sultana, R., Ferree, A., Smith, K., Barone, E., Perluigi, M., et al. (2012). Redox proteomics analyses of the influence of co-expression of wildtype or mutated LRRK2 and tau on C. elegans protein expression and oxidative modification: relevance to Parkinson disease. Antioxid. Redox Signal. 17, 14901506. doi: 10.1089 /ars.2011.4312

Ducas, V. C., and Rhoades, E. (2012). Quantifying interactions of beta-synuclein and gamma-synuclein with model membranes. J. Mol. Biol. 423, 528-539. doi: 10.1016/j.jmb.2012.08.008

Dumitriu, A., Pacheco, C. D., Wilk, J. B., Strathearn, K. E., Latourelle, J. C., Goldwurm, S., et al. (2011). Cyclin-G-associated kinase modifies alpha-synuclein expression levels and toxicity in Parkinson's disease: results from the GenePD Study. Hum. Mol. Genet. 20, 1478-1487. doi: 10.1093/hmg/ddr026

Dusonchet, J., Li, H., Guillily, M., Liu, M., Stafa, K., Derada, C., et al. (2014). A Parkinson's disease gene regulatory network identifies the signaling protein RGS2 as a modulator of LRRK2 activity and neuronal toxicity. Hum. Mol. Genet. doi: 10.1093/hmg/ddu202 [Epub ahead of print].

Dzamko, N., Deak, M., Hentati, F., Reith, A. D., Prescott, A. R., Alessi, D. R., et al. (2010). Inhibition of LRRK2 kinase activity leads to dephosphorylation of Ser(910)/Ser(935), disruption of 14-3-3 binding and altered cytoplasmic localization. Biochem. J. 430, 405-413. doi: 10.1042/BJ20100784

Dzamko, N., Inesta-Vaquera, F., Zhang, J., Xie, C., Cai, H., Arthur, S., et al. (2012). The IkappaB kinase family phosphorylates the Parkinson's disease kinase LRRK2 at Ser935 and Ser910 during Toll-like receptor signaling. PLoS ONE 7:e39132. doi: 10.1371/journal.pone.0039132

Faith, J. J., Hayete, B., Thaden, J. T., Mogno, I., Wierzbowski, J., Cottarel, G., et al. (2007). Large-scale mapping and validation of Escherichia coli transcriptional regulation from a compendium of expression profiles. PLoS Biol. 5:e8. doi: 10.1371/journal.pbio.0050008

Ferree, A., Guillily, M., Li, H., Smith, K., Takashima, A., Squillace, R., et al. (2012). Regulation of physiologic actions of LRRK2: focus on autophagy. Neurodegener. Dis. 10, 238-241. doi: 10.1159/000332599

Gallo, K. A., and Johnson, G. L. (2002). Mixed-lineage kinase control of JNK and p38 MAPK pathways. Nat. Rev. Mol. Cell Biol. 3, 663-672. doi: 10.1038/nrm906

Gasser, T., Hardy, J., and Mizuno, Y. (2011). Milestones in PD genetics. Mov. Disord. 26, 1042-1048. doi: $10.1002 / \mathrm{mds} .23637$

Gehrke, S., Imai, Y., Sokol, N., and Lu, B. (2010). Pathogenic LRRK2 negatively regulates microRNA-mediated translational repression. Nature 466, 637-641. doi: 10.1038/nature09191

Gloeckner, C. J., Schumacher, A., Boldt, K., and Ueffing, M. (2009). The Parkinson disease-associated protein kinase LRRK2 exhibits MAPKKK activity and phosphorylates MKK3/6 and MKK4/7, in vitro. J. Neurochem. 109, 959-968. doi: $10.1111 / j .1471-4159.2009 .06024 . x$

Greene, J. G. (2012). Current status and future directions of gene expression profiling in Parkinson's disease. Neurobiol. Dis. 45 76-82. doi: 10.1016/j.nbd.2010.10.022

Greggio, E., and Cookson, M. R. (2009). Leucine-rich repeat kinase 2 mutations and Parkinson's disease: three questions. ASN Neuro 1:e00002. doi: 10.1042/AN20090007

Greggio, E., Jain, S., Kingsbury, A., Bandopadhyay, R., Lewis, P., Kaganovich, A., et al. (2006). Kinase activity is required for the toxic effects of mutant LRRK2/dardarin. Neurobiol. Dis. 23, 329-341. doi: 10.1016/j.nbd.2006. 04.001

Habig, K., Gellhaar, S., Heim, B., Djuric, V., Giesert, F., Wurst, W., et al. (2013). LRRK2 guides the actin cytoskeleton at growth cones together with ARHGEF7 and Tropomyosin 4. Biochim. Biophys. Acta 1832, 2352-2367. doi: 10.1016/j.bbadis. 2013.09.009

Healy, D. G., Falchi, M., O'Sullivan, S. S., Bonifati, V., Durr, A., Bressman, S., et al. (2008). International, Phenotype, genotype, and worldwide genetic penetrance of LRRK2-associated Parkinson's disease: a case-control study. Lancet Neurol. 7, 583-590. doi: 10.1016/S1474-4422(08)70117-0

Ho, C. C., Rideout, H. J., Ribe, E., Troy, C. M., and Dauer, W. T. (2009). The Parkinson disease protein leucine-rich repeat kinase 2 transduces death signals via Fas-associated protein with death domain and caspase- 8 in a cellular model of neurodegeneration. J. Neurosci. 29, 1011-1016. doi: 10.1523/JNEUROSCI.517508.2009

Hsu, C. H., Chan, D., Greggio, E., Saha, S., Guillily, M. D., Ferree, A., et al. (2010a). MKK6 binds and regulates expression of Parkinson's disease-related protein LRRK2. J. Neurochem. 112, 1593-604. doi: 10.1111/j.1471-4159.2010. 06568.x

Hsu, C. H., Chan, D., and Wolozin, B. (2010b). LRRK2 and the stress response: interaction with MKKs and JNK-interacting proteins. Neurodegener. Dis. 7, 6875. doi: $10.1159 / 000285509$

Imai, Y., Gehrke, S., Wang, H. Q., Takahashi, R., Hasegawa, K., Oota, E., et al. (2008). Phosphorylation of 4E-BP by LRRK2 affects the maintenance of dopaminergic neurons in Drosophila. EMBO J. 27, 2432-2443. doi: 10.1038/emboj. 2008.163

Ito, G., Okai, T., Fujino, G., Takeda, K., Ichijo, H., Katada, T., et al. (2007). GTP binding is essential to the protein kinase activity of LRRK2, a causative gene product for familial Parkinson's disease. Biochemistry 46, 1380-1388. doi: 10.1021/ bi061960m 
Kelkar, N., Standen, C. L., and Davis, R. J. (2005). Role of the JIP4 scaffold protein in the regulation of mitogen-activated protein kinase signaling pathways. Mol. Cell. Biol. 25, 2733-2743. doi: 10.1128/MCB.25.7.2733-2743.2005

Ko, H. S., Bailey, R., Smith, W. W., Liu, Z., Shin, J. H., Lee, Y. I., et al. (2009). CHIP regulates leucine-rich repeat kinase-2 ubiquitination, degradation, and toxicity. Proc. Natl. Acad. Sci. U.S.A. 106, 2897-2902. doi: 10.1073/pnas.0810 123106

Kumar, A., Greggio, E., Beilina, A., Kaganovich, A., Chan, D., Taymans, J. M., et al. (2010). The Parkinson's disease associated LRRK2 exhibits weaker in vitro phosphorylation of 4E-BP compared to autophosphorylation. PLoS ONE 5:e8730. doi: 10.1371/journal.pone.0008730

Law, B. M., Spain, V. A., Leinster, V. H., Chia, R., Beilina, A., Cho, H. J., et al. (2014). A direct interaction between leucine-rich repeat kinase 2 and specific beta-tubulin isoforms regulates tubulin acetylation. J. Biol. Chem. 289, 895-908. doi: 10.1074/jbc.M113.507913

Lees, A. J., Hardy, J., and Revesz, T. (2009). Parkinson's disease. Lancet 373, 2055 2066. doi: 10.1016/S0140-6736(09)60492-X

Lewis, P. A., Greggio, E., Beilina, A., Jain, S., Baker, A., and Cookson, M. R. (2007). The R1441C mutation of LRRK2 disrupts GTP hydrolysis Biochem. Biophys. Res. Commun. 357, 668-671. doi: 10.1016/j.bbrc.2007. 04.006

Li, X., Tan, Y. C., Poulose, S., Olanow, C. W., Huang, X. Y., and Yue, Z. (2007). Leucine-rich repeat kinase 2 (LRRK2)/PARK8 possesses GTPase activity that is altered in familial Parkinson's disease R1441C/G mutants. J. Neurochem. 103, 238-247.

Liou, A. K., Leak, R. K., Li, L., and Zigmond, M. J. (2008). Wild-type LRRK2 but not its mutant attenuates stress-induced cell death via ERK pathway. Neurobiol. Dis. 32, 116-124. doi: 10.1016/j.nbd.2008.06.016

Liu, Z., Lee, J., Krummey, S., Lu, W., Cai, H., and Lenardo, M. J. (2011). The kinase LRRK2 is a regulator of the transcription factor NFAT that modulates the severity of inflammatory bowel disease. Nat. Immunol. 12, 1063-1070. doi: 10.1038/ni.2113

Liu, Z., Wang, X., Yu, Y., Li, X., Wang, T., Jiang, H., et al. (2008). A Drosophila model for LRRK2-linked parkinsonism. Proc. Natl. Acad. Sci. U.S.A. 105, 2693-2698. doi: 10.1073/pnas.0708452105

Macleod, D., Dowman, J., Hammond, R., Leete, T., Inoue, K., and Abeliovich, A. (2006). The familial parkinsonism gene LRRK2 regulates neurite process morphology. Neuron 52, 587-593. doi: 10.1016/j.neuron.2006. 10.008

Marin, I. (2008). Ancient origin of the Parkinson disease gene LRRK2. J. Mol. Evol. 67, 41-50. doi: 10.1007/s00239-008-9122-4

Martin, I., Kim, J. W., Lee, B. D., Kang, H. C., Xu, J. C., Jia, H., et al. (2014). Ribosomal protein s15 phosphorylation mediates LRRK2 neurodegeneration in Parkinson's disease. Cell 157, 472-485. doi: 10.1016/j.cell.2014. 01.064

Matta, S., Van Kolen, K., da Cunha, R., van den Bogaart, G., Mandemakers, W., Miskiewicz, K., et al. (2012). LRRK2 controls an EndoA phosphorylation cycle in synaptic endocytosis. Neuron 75, 1008-1021. doi: 10.1016/j.neuron.2012.08.022

Mazzulli, J. R., Xu, Y. H., Sun, Y., Knight, A. L., McLean, P. J., Caldwell, G. A., et al. (2011). Gaucher disease glucocerebrosidase and alpha-synuclein form a bidirectional pathogenic loop in synucleinopathies. Cell 146, 37-52. doi: 10.1016/j.cell.2011.06.001

Muda, K., Bertinetti, D., Gesellchen, F., Hermann, J. S., von Zweydorf, F., Geerlof, A., et al. (2014). LRRK2 mutation R1441C/G/H impairs PKA phosphorylation of LRRK2 and disrupts its interaction with 14-3-3. Proc. Natl. Acad. Sci. U.S.A. 111, E34-E43. doi: 10.1073/pnas.1312701111

Narendra, D. P., Jin, S. M., Tanaka, A., Suen, D. F., Gautier, C. A., Shen, J., etal. (2010). PINK1 is selectively stabilized on impaired mitochondria to activate Parkin. PLoS Biol. 8:e1000298. doi: 10.1371/journal.pbio. 1000298

Nichols, R. J., Dzamko, N., Morrice, N. A., Campbell, D. G., Deak, M., Ordureau, A., et al. (2010). 14-3-3 binding to LRRK2 is disrupted by multiple Parkinson's disease-associated mutations and regulates cytoplasmic localization. Biochem. J. 430, 393-404. doi: 10.1042/BJ20100483

Pankratz, N., Wilk, J. B., Latourelle, J. C., DeStefano, A. L., Halter, C., Pugh, E. W., et al. (2009). Molecular genetic, genomewide association study for susceptibility genes contributing to familial Parkinson disease. Hum. Genet. 124, 593-605. doi: 10.1007/s00439-008-0582-9
Parisiadou, L., Xie, C., Cho, H. J., Lin, X., Gu, X. L., Long, C. X., et al. (2009). Phosphorylation of ezrin/radixin/moesin proteins by LRRK2 promotes the rearrangement of actin cytoskeleton in neuronal morphogenesis. J. Neurosci. 29, 13971-13980. doi: 10.1523/JNEUROSCI.3799-09.2009

Parisiadou, L., Yu, J., Sgobio, C., Xie, C., Liu, G., Sun, L., et al. (2014). LRRK2 regulates synaptogenesis and dopamine receptor activation through modulation of PKA activity. Nat. Neurosci. 17, 367-376. doi: 10.1038/ nn.3636

Perlmutter, J. D., Braun, A. R., and Sachs, J. N. (2009). Curvature dynamics of alpha-synuclein familial Parkinson disease mutants: molecular simulations of the micelle- and bilayer-bound forms. J. Biol. Chem. 284, 7177-7189. doi: 10.1074/jbc.M808895200

Plowey, E. D., Cherra, S. J. III, Liu, Y. J., and Chu, C. T. (2008). Role of autophagy in G2019S-LRRK2-associated neurite shortening in differentiated SH-SY5Y cells. J. Neurochem. 105, 1048-1056. doi: 10.1111/j.1471-4159.2008. 05217.x

Plun-Favreau, H., Klupsch, K., Moisoi, N., Gandhi, S., Kjaer, S., Frith, D., et al. (2007). The mitochondrial protease HtrA2 is regulated by Parkinson's disease-associated kinase PINK1. Nat. Cell Biol. 9, 1243-1252. doi: 10.1038/ ncb1644

Saha, S., Guillily, M. D., Ferree, A., Lanceta, J., Chan, D., Ghosh, J., et al. (2009). LRRK2 modulates vulnerability to mitochondrial dysfunction in Caenorhabditis elegans. J. Neurosci. 29, 9210-9218. doi: 10.1523/JNEUROSCI.228109.2009

Samann, J., Hegermann, J., von Gromoff, E., Eimer, S., Baumeister, R., and Schmidt, E. (2009). Caenorhabditits elegans LRK-1 and PINK-1 act antagonistically in stress response and neurite outgrowth. J. Biol. Chem. 284, 16482-16491. doi: 10.1074/jbc.M808255200

Sancho, R. M., Law, B. M., and Harvey, K. (2009). Mutations in the LRRK2 RocCOR tandem domain link Parkinson's disease to Wnt signalling pathways. Hum. Mol. Genet. 18, 3955-3968. doi: 10.1093/hmg/ddp337

Shin, N., Jeong, H., Kwon, J., Heo, H. Y., Kwon, J. J., Yun, H. J., et al. (2008). LRRK2 regulates synaptic vesicle endocytosis. Exp. Cell Res. 314, 2055-2065. doi: 10.1016/j.yexcr.2008.02.015

Simon-Sanchez, J., Herranz-Perez, V., Olucha-Bordonau, F., and Perez-Tur, J. (2006). LRRK2 is expressed in areas affected by Parkinson's disease in the adult mouse brain. Eur. J. Neurosci. 23, 659-666. doi: 10.1111/j.1460-9568.2006. 04616.x

Skibinski, G., Nakamura, K., Cookson, M. R., and Finkbeiner, S. (2014). Mutant LRRK2 toxicity in neurons depends on LRRK2 levels and synuclein but not kinase activity or inclusion bodies. J. Neurosci. 34, 418-433. doi: 10.1523/JNEUROSCI.2712-13.2014

Smith, W. W., Pei, Z., Jiang, H., Moore, D. J., Liang, Y., West, A. B., et al. (2005). Leucine-rich repeat kinase 2 (LRRK2) interacts with parkin and mutant LRRK2 induces neuronal degeneration. Proc. Natl. Acad. Sci. U.S.A. 102, 18676-18681. doi: 10.1073/pnas. 0508052102

Stafa, K., Trancikova, A., Webber, P. J., Glauser, L., West, A. B., and Moore, D. J. (2012). GTPase activity and neuronal toxicity of Parkinson's diseaseassociated LRRK2 is regulated by ArfGAP1. PLoS Genet. 8:e1002526. doi: 10.1371/journal.pgen.1002526

Taylor, J. P., Mata, I. F., and Farrer, M. J. (2006). LRRK2: a common pathway for parkinsonism, pathogenesis and prevention? Trends Mol. Med. 12, 76-82. doi: 10.1016/j.molmed.2005.12.004

Tong, Y., Yamaguchi, H., Giaime, E., Boyle, S., Kopan, R., Kelleher, R. J. III, et al. (2010). Loss of leucine-rich repeat kinase 2 causes impairment of protein degradation pathways, accumulation of alpha-synuclein, and apoptotic cell death in aged mice. Proc. Natl. Acad. Sci. U.S.A. 107, 9879-9884. doi: 10.1073/pnas. 1004676107

Usenovic, M., Knight, A. L., Ray, A., Wong, V., Brown, K. R., Caldwell, G. A., et al. (2012). Identification of novel ATP13A2 interactors and their role in alpha-synuclein misfolding and toxicity. Hum. Mol. Genet. 21, 3785-3794. doi: $10.1093 / \mathrm{hmg} / \mathrm{dds} 206$

Varkey, J., Isas, J. M., Mizuno, N., Jensen, M. B., Bhatia, V. K., Jao, C. C., et al. (2010). Membrane curvature induction and tubulation are common features of synucleins and apolipoproteins. J. Biol. Chem. 285, 32486-32493. doi: 10.1074/jbc.M110.139576

Venderova, K., Kabbach, G., Abdel-Messih, E., Zhang, Y., Parks, R. J., Imai, Y., et al. (2009). Leucine-rich repeat kinase 2 interacts with Parkin, DJ-1 and PINK-1 in 
a Drosophila melanogaster model of Parkinson's disease. Hum. Mol. Genet. 18, 4390-4404. doi: 10.1093/hmg/ddp394

Verhey, K. J., Meyer, D., Deehan, R., Blenis, J., Schnapp, B. J., Rapoport, T. A., et al. (2001). Cargo of kinesin identified as JIP scaffolding proteins and associated signaling molecules. J. Cell Biol. 152, 959-970. doi: 10.1083/jcb.152. 5.959

Wang, D., Tang, B., Zhao, G., Pan, Q., Xia, K., Bodmer, R., et al. (2008). Dispensable role of Drosophila ortholog of LRRK2 kinase activity in survival of dopaminergic neurons. Mol. Neurodegener. 3:3. doi: 10.1186/17501326-3-3

Wang, L. H., Besirli, C. G., and Johnson, E. M. Jr. (2004). Mixed-lineage kinases: a target for the prevention of neurodegeneration. Annu. Rev. Pharmacol. Toxicol. 44, 451-474. doi: 10.1146/annurev.pharmtox.44.101802. 121840

West, A. B., Moore, D. J., Biskup, S., Bugayenko, A., Smith, W. W., Ross, C. A., et al. (2005). Parkinson's disease-associated mutations in leucine-rich repeat kinase 2 augment kinase activity. Proc. Natl. Acad. Sci. U.S.A. 102, 16842-16847. doi: 10.1073/pnas.0507360102

Whitmarsh, A. J. (2006). The JIP family of MAPK scaffold proteins. Biochem. Soc. Trans. 34, 828-832. doi: 10.1042/BST0340828

Wszolek, Z. K., Pfeiffer, R. F., Tsuboi, Y., Uitti, R. J., McComb, R. D., Stoessl, A. J., etal. (2004). Autosomal dominant parkinsonism associated with variable synuclein and tau pathology. Neurology 62, 1619-1622. doi: 10.1212/01.WNL.0000125015.06989.DB

Xiong, Y., Yuan, C., Chen, R., Dawson, T. M., and Dawson, V. L. (2012). ArfGAP1 is a GTPase activating protein for LRRK2: reciprocal regulation of ArfGAP1 by LRRK2. J. Neurosci. 32, 3877-3886. doi: 10.1523/JNEUROSCI.4566-11.2012

Yang, S. H., Sharrocks, A. D., and Whitmarsh, A. J. (2013). MAP kinase signalling cascades and transcriptional regulation. Gene 513, 1-13. doi: 10.1016/j.gene. 2012.10 .033
Yao, C., El Khoury, R., Wang, W., Byrd, T. A., Pehek, E. A., Thacker, C., etal. (2010). LRRK2-mediated neurodegeneration and dysfunction of dopaminergic neurons in a Caenorhabditis elegans model of Parkinson's disease. Neurobiol. Dis. 40, 73-81. doi: 10.1016/j.nbd.2010. 04.002

Yuan, Y., Cao, P., Smith, M. A., Kramp, K., Huang, Y., Hisamoto, N., et al. (2011). Dysregulated LRRK2 signaling in response to endoplasmic reticulum stress leads to dopaminergic neuron degeneration in C. elegans. PLoS ONE 6:e22354. doi: 10.1371/journal.pone.0022354

Zimprich, A., Biskup, S., Leitner, P., Lichtner, P., Farrer, M., Lincoln, S., et al. (2004). Mutations in LRRK2 cause autosomal-dominant parkinsonism with pleomorphic pathology. Neuron 44, 601-607. doi: 10.1016/j.neuron.2004.11.005

Conflict of Interest Statement: Benjamin Wolozin has a conflict of interest based on ownership of equity in Aquinnah Pharmaceuticals, Inc., which is a company focusing on neurodegenerative diseases. Joon Y. Boon, Chelsea Trengrove, and Julien Dusonchet have no conflicts of interest.

Received: 07 April 2014; paper pending published: 24 May 2014; accepted: 19 June 2014; published online: 09 July 2014

Citation: Boon JY, Dusonchet J, Trengrove C and Wolozin B (2014) Interaction of LRRK2 with kinase and GTPase signaling cascades. Front. Mol. Neurosci. 7:64. doi: 10.3389/fnmol.2014.00064

This article was submitted to the journal Frontiers in Molecular Neuroscience.

Copyright (C) 2014 Boon, Dusonchet, Trengrove and Wolozin. This is an open-access article distributed under the terms of the Creative Commons Attribution License (CC BY). The use, distribution or reproduction in other forums is permitted, provided the original author(s) or licensor are credited and that the original publication in this journal is cited, in accordance with accepted academic practice. No use, distribution or reproduction is permitted which does not comply with these terms. 